\title{
Pain as a challenge in nursing home residents with behavioral and psychological symptoms of dementia
}

This article was published in the following Dove Press journal:

Clinical Interventions in Aging

\author{
Tomasz Nowak' \\ Agnieszka Neumann- \\ Podczaska ${ }^{2}$ \\ Ewa Deskur-Śmielecka' \\ Arkadiusz Styszyński \\ Katarzyna Wieczorowska- \\ Tobis' \\ 'Laboratory of Geriatrics, \\ Department of Palliative Medicine, \\ Poznan University of Medical Sciences, \\ Poznań, Poland; ${ }^{2}$ Department of \\ Geriatric Medicine and Gerontology, \\ Poznan University of Medical Sciences, \\ Poznań, Poland
}

Introduction: In patients with dementia, observational scales are recommended for use in the assessment of pain. Unfortunately, their application is rare, and as a consequence pain is frequently underdiagnosed and undertreated in these types of subjects. Thus, the aim of the study was to assess analgesic treatment in nursing home residents with cognitive impairment and to delineate the relationship between pain and behavioral and psychological symptoms of dementia.

Patients and methods: The research was conducted in 2 nursing home facilities in Wielkopolska, Poland. The analyzed group consisted of 96 residents ( 78 female) with moderate and severe cognitive impairment in whom pain was assessed with the Abbey Pain Scale (APS) and agitation with the Cohen-Mansfield Agitation Inventory (CMAI). Thereafter, medical files related to drug prescriptions were analyzed.

Results: Analgesics were consumed by 33 individuals (34\%); 24 (25\%) received regular pain treatment and 7 individuals $(7 \%)$ - as when needed pain treatment. A relationship was found between the APS and CMAI $(r=0.45, p<0.0001)$. Subjects with a higher CMAI received sedative drugs more frequently $(p<0.001)$, and despite having a higher APS $(p=0.001)$, this did not correlate with higher analgesia.

Conclusion: Our study suggests that pain can be an important underlying cause of behavioral disturbances in older subjects with dementia. In order to reduce their frequency and to avoid excessive usage of sedatives, proper pain assessment and management are essential.

Keywords: pain, analgesics, nursing home residents, older individuals, Abbey Pain Scale, CMAI

\section{Introduction}

Dementia affects approximately 24.3 million people worldwide and is expected to increase to 115 million by 2050 as the population of older individuals grows. ${ }^{1}$ Approximately one-third of people with dementia are nursing home residents (NHR).

Advanced age correlates not only with the increased prevalence of dementia, but also as a result of other comorbidities leading to pain. ${ }^{2}$ The data regarding the pain frequency among NHR with dementia are unclear, but it is estimated that $40 \%-60 \%$ of them suffer daily from moderate to severe pain..$^{3-5}$

Dementia is frequently accompanied by behavioral and psychological symptoms of dementia (BPSD) defined as signs and symptoms of disturbed perception, thought content, mood, or behavior. ${ }^{6}$ In patients with BPSD, due to language, memory, and abstractthinking deficits, pain self-report capacity is substantially decreased or even absent. In such patients, observational pain scales need to be used. ${ }^{7}$ However, their application may be a challenge as the estimation of pain depends not only on observation and communication with the patient but also on the knowledge of caregivers and medical staff and their ability to make an accurate diagnosis. ${ }^{8}$ As a result, pain in elderly subjects with dementia is frequently underdiagnosed, and thus undertreated. ${ }^{4}$ 
There is growing evidence that behavioral disturbances (ie, agitation and aggression) are common pain behaviors in patients with impaired communication skills. Consequently, those who experience them are inappropriately prescribed antipsychotic medication. ${ }^{9}$ For these drugs, the risk-benefit ratio in patients with cognitive impairment is questioned due to side effects and potential negative health consequences. According to the report of the Department of Health in UK, 1,620 excess strokes and 1,800 deaths were observed yearly among 180,000 people with dementia who were prescribed antipsychotics. ${ }^{10}$ These data show that in patients with dementia the key issue is to find safer and effective methods to reduce agitation and aggression. More effective diagnosis of pain as well as treatment may contribute to the overall prevention of agitation, and thus lower the usage of antipsychotic drugs. ${ }^{10}$

The aim of the study was to assess analgesic use among NHR with moderate to severe cognitive impairment and to delineate the relationship between pain and behavioral disturbances.

\section{Patients and methods}

The project was approved by the Bioethical Committee of Karol Marcinkowski University of Medical Sciences in Poznan, Poland.

\section{Participants}

The research was conducted in 2 nursing home facilities in Wielkopolska region of Poland. All NHR aged 65 years and above were screened for cognitive impairment. Only those with at least moderate cognitive impairment participated in the study.

All subjects gave their written informed consent after receiving a full explanation of the nature of the study. In the case of patients lacking capacity to provide informed consent, the consent of a legal representative was required.

\section{Study procedure}

In all studied subjects, the assessment of activities of daily living (ADLs), pain, and agitation were performed by trained nurses employed by the nursing home, who had knowledge of the patient. Thereafter, medical files related to drug prescriptions were analyzed.

\section{Assessments of ADL}

ADL was assessed with the Barthel Index. ${ }^{11}$ The scale consists of 10 variables describing ADL and mobility (eg, toileting, bathing, eating, dressing, continence, transfers, and ambulation). Total possible scores range from 0 to 100 , with lower scores indicating increased disability.

Subjects receive numerical scores based on whether they require physical assistance to perform the task or can complete it independently. Items were weighted according to the professional judgment of the trained nurse having knowledge of the patient. A subject scoring 0 points would be dependent in all assessed ADL, whereas a score of 100 would reflect independence in these. The Barthel Index has demonstrated high interrater reliability and test-retest reliability as well as high correlations with other measures of physical disability. ${ }^{11}$

\section{Cognitive screening}

Cognitive functions were assessed with the Abbreviated Mental Test Score (AMTS). ${ }^{12}$ The test is composed of 10 questions; each correctly answered question is scored with 1 point. The maximum score is 10 points. A score above 6 points implies normal cognition, 4- 6 points - the presence of moderate cognitive impairment, and 0-3 points - severe cognitive impairment.

AMTS could successfully differentiate the dementia group from the nondementia group. AMTS is a valid cognitive assessment tool and can be used for dementia screening. ${ }^{13}$

\section{Pain assessment}

The presence of pain was evaluated by a trained nurse with the Abbey Pain Scale (APS). ${ }^{14}$ The instrument is designed to assist in the assessment of pain in patients who are unable to clearly articulate their needs. APS is an observational tool which should be used as a movement-based assessment. The staff recording the scale observe the patient while they are being moved, eg, during pressure area care, while showering, etc. The scale consists of 6 parts describing patient's behavior: vocalization, facial expression, change in body language, behavioral change, physiological changes, and physical changes. Each part has 3 levels of intensity. The maximum score is 18 points, a score of 14 and more refers to severe pain, $8-13$ points - moderate pain, 3-7 - mild pain, and $0-2$ corresponds to no pain. Validity and internal reliability of APS were found to be satisfactory. Qualitative evidence gathered from users of the scale indicated that APS is useful clinical device. ${ }^{15}$

\section{Agitation assessment}

Agitation was assessed using the Cohen-Mansfield Agitation Inventory (CMAI). The inventory assesses the frequency of 29 behavior characteristics for agitation. Each item is rated on 
a 7-point scale of frequency ( $1=$ not present, $7=$ several times an hour). The final results range from 29 to 203 points. ${ }^{16}$

CMAI demonstrates high internal consistency. However, the interrrater reliability is adequate, the correlation of CMAI with the Behavioral Syndromes Scale for Dementia and the Behavioral Pathology in Alzhemer's Disease was found. It means that CMAI is a valid measure of agitation in NHR. ${ }^{17}$

Based on the CMAI results, the NHR were divided into 2 subgroups as follows: ${ }^{18}$

Group $1-<39$ points - subjects without significant behavioral disturbances.

Group 2-39 and above - subjects with significant behavioral disturbances.

\section{Analysis of pharmacotherapy}

For the purpose of the qualitative and quantitative analysis, all medicines were coded based on the Anatomical Therapeutic Chemical classification system, recommended by The World Health Organization, and their number was calculated. ${ }^{19}$ Analgesics were divided according to the analgesic ladder (step I - nonopioids [acetaminophen, nonsteroidal anti-inflammatory drugs \{NSAIDs\}], step II - weak opioids, step III - strong opioids; various drug combinations [under 1 therapeutic formula: acetaminophen/tramadol and under numerous therapeutic formulas]). Prevalence of analgesics and sedatives (defined as benzodiazepines, neuroleptics [both conventional and atypical], and hydroxyzine) was analyzed. Analgesics consumption was divided into 3 categories: regular use, use as needed (pro re nata [PRN]), and their combination. Maximum allowed daily dosages of all analgesics were defined according to official product characteristics. ${ }^{20}$

\section{Statistical analysis}

All results are expressed as the mean (SD) and median (range). To evaluate the normality of distribution of the variables, the Shapiro-Wilk test was applied. As most of the investigated variables were not normally distributed, nonparametric Mann-Whitney test was employed. Betweengroup differences were assessed by Fisher's exact test due to small sample size. The correlation between APS and CMAI was analyzed with Spearman's rank correlation coefficient. Statistical significance was set at $p<0.05$ for all analyses.

\section{Results}

\section{Characteristics of the study group}

The analyzed group consisted of 96 residents; among them, 78 were female ( $81 \%$ study group).
The mean age of studied subjects was 84.0 (5.9) years; median: 83 (75-102 years); among them only 18 (19\%) were aged 65-74 years. The mean length of institutionalization was 55.0 (48.4) months, median 38.5 (1-222 months); 4 individuals (4\%) had a nursing home stay of less than 3 months, while 11 (11\%) resided longer than 10 years.

Analyzed subjects were very dependent as 63 of them $(66 \%)$ had a Barthel Index $<20$ points - mean: 25.0 (27.8 points), median: 10.0 (5-95 points). Moderate cognitive impairment (4-6 points in AMTS scale) was observed in 17 individuals (18\%), while severe ( $0-3$ points in AMTS) was seen in 79 subjects $(82 \%)$ - mean: $1.0(1,9)$; median: $0(0-6)$.

The mean number of drugs consumed by studied individuals was 5.5 (3.0), median: $5(0-13)$. Only 1 person (1\%) did not receive any drugs, and 13 people received 10 or more medications. Sedative drugs were consumed by 63 (66\%) subjects; among them, 25 (40\%) had more than 1 drug with a sedative effect -1 individual was even prescribed 4 sedative medications at 1 time. Benzodiazepines were consumed by 26 subjects $(27 \% ; 1$ person received 2 benzodiazepines simultaneously); neuroleptics by 45 (47\%; 9 persons received 2 neuroleptics concomitantly); and hydroxyzine by $12(19 \%)$.

\section{Characteristics of pain treatment}

Table 1 shows the detailed distribution of routine pain treatment in particular subjects. There were 3 subjects who experienced severe pain and were treated with substantially lower than the maximum allowed daily dosages of analgesics.

One-third of studied individuals (33 persons; 34\%) consumed analgesics. Among them, 24 (25\%) received routine pain treatment, 7 as needed (PRN) and $2(2 \%)$ as both regular and as needed (PRN) schemes of treatment.

In regard to nonopioid analgesia (acetaminophen or NSAIDs) 13 persons (14\%) received them regularly as monotherapy (acetaminophen - 10 individuals and NSAIDs 3 individuals). The maximum consumed daily dosage for acetaminophen was $1,500 \mathrm{mg}$, but most subjects received $500 \mathrm{mg}$ daily (Table 1 ).

No one received routine monotherapy with analgesics from the second step of the analgesic ladder, while $7(7 \%)$ individuals received routine combination preparations consisting of tramadol and acetaminophen in 1 therapeutic formula.

Only $2(2 \%)$ subjects received a strong opioid (buprenorphine) as monotherapy.

Combinations of either opioids or nonopioids were used in 11 subjects (11\%) (as separate formulations). 


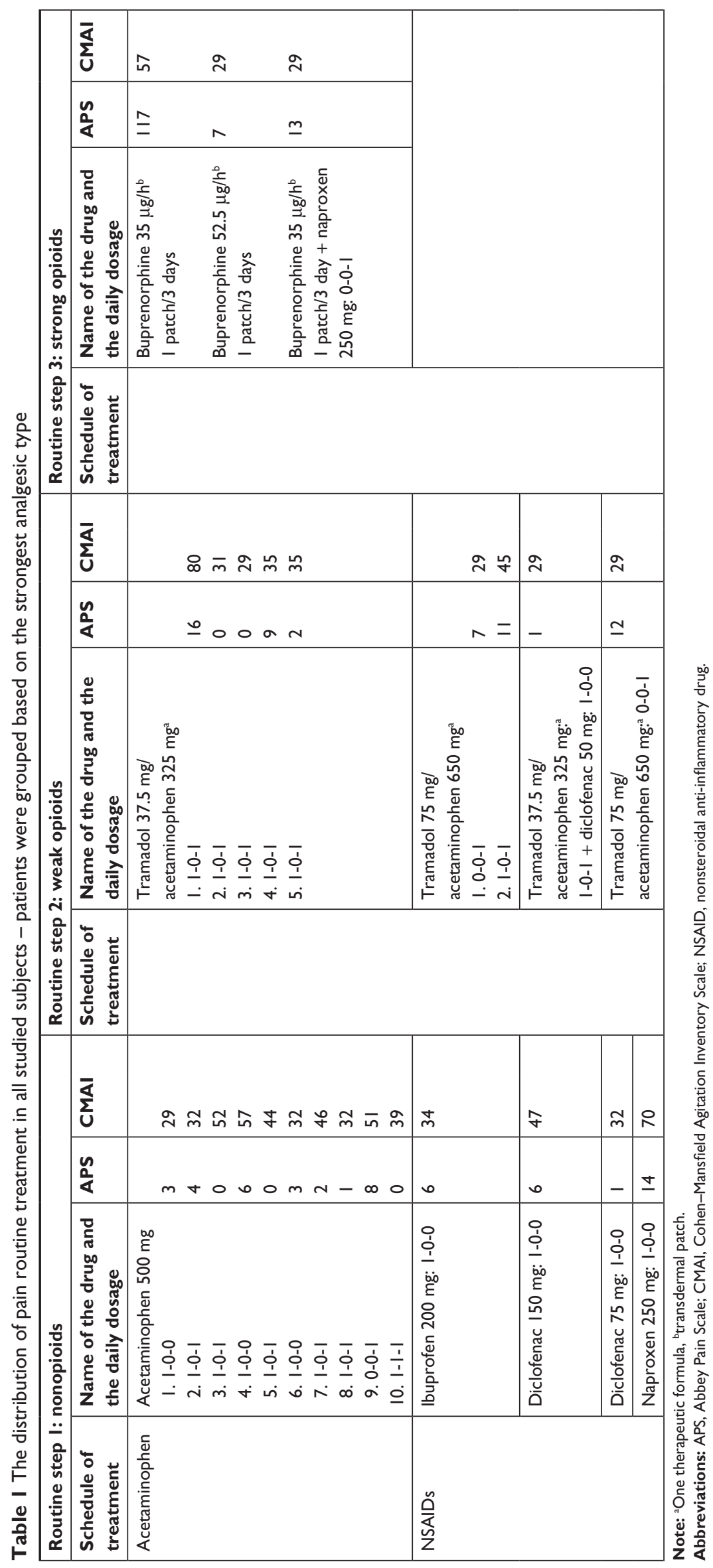




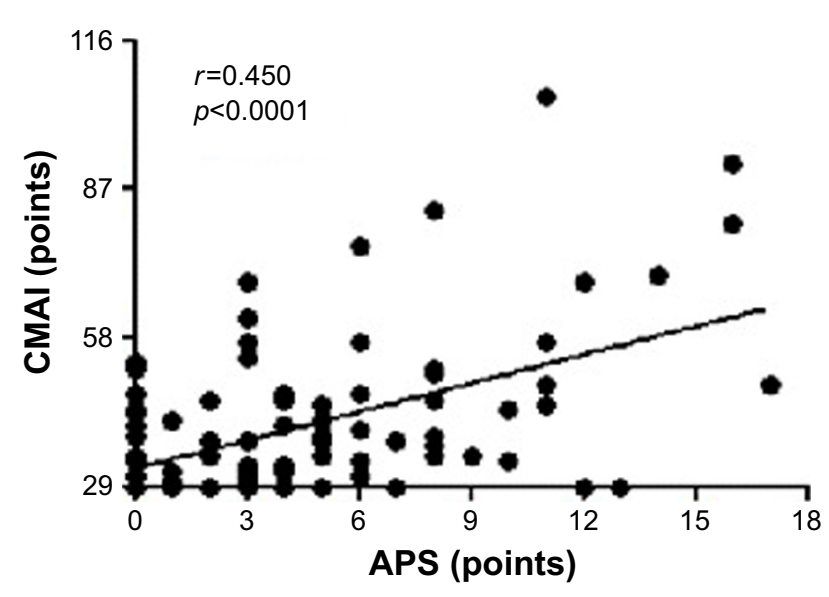

Figure I Relationship between pain and behavioral disturbances, $\mathrm{N}=96$. Abbreviations: APS, Abbey Pain Scale; CMAI, Cohen-Mansfield Agitation Inventory Scale.

Additionally, 2 (2\%) subjects received regular treatment with combinations of drugs from different steps of the analgesic ladder.

\section{Relationship between pain and behavioral disturbances}

The average result in the APS was 4.0 (4.3) points, median: 3 (0-17 points). The average CMAI score was 40 (15) points, median: 35 (29-105 points).

A relationship was found between the results obtained by the participants in the APS and the CMAI $(r=0.45, p<0.0001$; Figure 1).
Table 2 shows the detailed characteristic of studied subjects divided into 2 groups based on the CMAI. Importantly, subjects with a higher CMAI received sedative drugs more frequently $(p<0.001)$. They had a higher APS $(p=0.001)$, but they did not receive analgesics more often than those with a lower CMAI (Table 2).

\section{Discussion}

Diagnosing pain in subjects with dementia is a challenging problem as they are incapable of expressing pain in typical ways. Thus pain is often inappropriately undertreated in these individuals. Indeed, we found that among NHR with moderate and severe cognitive impairment only $30 \%$ were treated with analgesics, with the majority receiving medication from the first step of the analgesic ladder. Among them, only a few received opioids still experienced mild to moderate pain according to APS.

Our results are similar to those of Closs et $\mathrm{al}^{21}$ who revealed that $32 \%$ of NHR with moderate to severe cognitive impairment were treated with nonopioids while only $6 \%$ were treated with opioids. In the study of Monroe et al, ${ }^{22}$ the prescription of analgesics was even lower, as only $20 \%$ of NHR with dementia had them prescribed. However, due to the challenging issue of methodological discrepancies, it is difficult to compare inadequate pain treatment in different studies as large between-countries differences in analgesics use exist. For example, in the study of Husebo et al, ${ }^{23}$ as many

Table 2 The characteristics of studied subjects divided based on CMAI

\begin{tabular}{|c|c|c|c|}
\hline & $\begin{array}{l}\text { Group I (n=57) } \\
\text { Mean (SD) } \\
\text { Median (range) }\end{array}$ & $\begin{array}{l}\text { Group } 2(n=39) \\
\text { Mean (SD) } \\
\text { Median (range) }\end{array}$ & $p$-value \\
\hline \multirow[t]{2}{*}{ Age (years) } & $83.3(9.0)$ & $84.8(8.3)$ & ns \\
\hline & $85(65-104)$ & $86(65-98)$ & \\
\hline Gender (Males) & II & 7 & ns \\
\hline \multirow[t]{2}{*}{ Length of institutionalization (months) } & $63.5(54.8)$ & $42.7(35.0)$ & ns \\
\hline & $52(I-222)$ & $32(6-162)$ & \\
\hline \multirow[t]{2}{*}{ Barthel scale } & $23.9(28.1)$ & $26.2(28.0)$ & ns \\
\hline & $10(5-95)$ & $10(5-95)$ & \\
\hline \multirow[t]{2}{*}{ AMTS } & I.I (I.9) & $\mathrm{I} .2(2.0)$ & ns \\
\hline & $0(0-6)$ & $0(0-6)$ & \\
\hline \multirow[t]{2}{*}{ APS } & $3.0(3.3)$ & $6.2(4.9)$ & $p<0.0$ I \\
\hline & $2(0-13)$ & $6(0-17)$ & \\
\hline \multirow[t]{2}{*}{ Number of drugs used } & $6.0(3.0)$ & $6.0(2.6)$ & ns \\
\hline & $6(0-13)$ & $6(1-13)$ & \\
\hline Number of subjects receiving routine analgesics ${ }^{\mathrm{a}}$ & 15 & II & ns \\
\hline Number of subjects receiving sedatives & 30 & 33 & $p<0.001$ \\
\hline Number of subjects receiving benzodiazepines & 9 & 17 & $p<0.001$ \\
\hline Number of subjects receiving neuroleptics & 24 & 21 & ns \\
\hline Number of subjects receiving hydroxyzine & 5 & 7 & ns \\
\hline
\end{tabular}

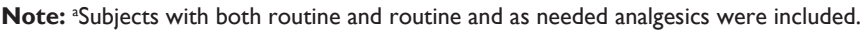

Abbreviations: AMTS, Abbreviated Mental Test Score; APS, Abbey Pain Scale; CMAI, Cohen-Mansfield Agitation Inventory Scale; ns, not significant. 
as $40 \%$ of NHR diagnosed with dementia received nonopioid analgesics and $18 \%$ opioids, but the study was limited to only those with major behavioral disturbances.

Interestingly, in our previous retrospective study of pain treatment in NHR, we showed that less than $15 \%$ of those with moderate to severe cognitive impairment were treated for pain. ${ }^{24} \mathrm{~A}$ higher percentage of treated subjects in our current study may suggest that the phenomenon of pain undertreatment in Poland may be on a downward trend due to enhanced efforts regarding education of medical staff on both proper pain diagnosis and treatment. A similar tendency was also observed by Sandvik et $\mathrm{al}^{25}$ who showed that the analgesic drug prescription increased significantly from 2000 to 2011 , especially the use of paracetamol and strong opioids. These authors also highlighted a possible change from underprescription of analgesic medication in people with dementia to an equal amount compared with patients without cognitive impairment. Olsen et al, ${ }^{26}$ who conducted a research among intensive care unit patients, also showed that the implementing a pain management algorithm decreased the length of intensive care unit stay and the duration of ventilation.

We found that even in individuals treated with analgesics, pain undertreatment may still be suspected based on the APS. This may be related to low dosages of analgesic drugs. As far as acetaminophen was concerned, no one received the maximum allowed daily dose, and almost 1 in every 2 received only $500 \mathrm{mg}$ of acetaminophen daily. This finding is consistent with the study of Husebo et al, ${ }^{23}$ which showed that $69 \%$ of NHR with dementia and behavioral disturbances consumed either no analgesics or only a low dose of acetaminophen.

As dementia impairs the ability to interpret and verbalize the pain experience, subjects with cognitive impairment may manifest pain through disruptive behaviors. We found a positive correlation between pain assessed based on the APS and agitation measured by CMAI. Similar results were obtained by other authors. ${ }^{27-29}$ Pain-related behavioral disturbances may be misinterpreted as behavioral and psychological symptoms of dementia, leading to excessive and inappropriate prescription of psychotropic medication. These drugs (ie, benzodiazepines and neuroleptics) in older individuals have been associated with impaired cognition, falls, fractures, and increased risk of death. ${ }^{30}$ We showed that subjects with major behavioral disturbances received more sedatives (mainly more benzodiazepines), and although their APS results were substantially higher they were not receiving more analgesia. Thus, one can speculate that in our cohort, inadequately managed pain as a potential factor for agitation and disruptive behavior was not taken into account.

\section{Limitations}

Our study also has some limitations such as the fact that the analyzed group was relatively small. We included, however, all residents living in 2 nursing home screened as having moderate or severe cognitive impairment based on AMTS. Thus, the group is nonselective, which is a strength of the study. In our analysis, coanalgesics were not included in the criteria of pain treatment. Due to the heterogeneity of different classes of coanalgesics, some of them could be prescribed due to other comorbidities, not necessarily for the treatment of pain; a similar approach has also been applied by other authors. ${ }^{22-24,27,28}$

Although we showed the association between pain and disruptive behaviors measured with CMAI, we did not take into account other potential causes of disruptive behaviors such as constipation, depression, or delirium. However, these disorders could also influence CMAI results, but their significance seems to be lower as other authors emphasize only the importance of pain management in patients with BPSD. ${ }^{21}$ Also, the psychometric properties of CMAI underline link between aggressive behaviors and pain. ${ }^{31}$

In summary, the pain assessment in cognitively impaired subjects seems to be a challenging process. Comprehensive pain assessment using observational pain scales should be applied in NHR with dementia. Moreover, there is evidence that a systematic approach to pain management by using a stepwise protocol for analgesia effectively reduces agitation in elderly NHR and can thus be an effective strategy to reduce the unnecessary use of psychotropic medicines. ${ }^{23,32,33}$

\section{Conclusion}

Our study suggests that pain can be an important underlying cause of behavioral disturbances in elderly subjects with dementia. In order to reduce these disruptive behaviors as well as to avoid excessive usage of sedatives, proper pain assessment and management are essential.

\section{Acknowledgment}

This project was supported by funding from the National Science Centre (Kraków, Poland) grant no. N N405 674340.

\section{Disclosure}

The authors report no conflicts of interest in this work.

\section{References}

1. World Health Organization (WHO), Alzheimer's Disease International. Dementia: A Public Health Priority. Geneva: WHO; 2012. 
2. Achterberg WP, Pot AM, Scherder EJ, Ribbe MW. Pain in the nursing home: assessment and treatment on different types of care wards. J Pain Symptom Manage. 2007;34(5):480-487.

3. Erdal A, Flo E, Selbaek G, et al. Associations between pain and depression in nursing home patients at different stages of dementia. $J$ Affect Disord. 2017;218:8-14.

4. Achterberg WP, Gambassi G, Finne-Soveri H, et al. Pain in European long-term care facilities: cross-national study in Finland, Italy and The Netherlands. Pain. 2010;148(1):70-74.

5. Achterberg WP, Pieper MJC, van Dalen-Kok AH, et al. Pain management in patients with dementia. Clin Interv Aging. 2013;8:1471-1482.

6. Kales HC, Gitlin LN, Lyketsos CG. Assessment and management of behavioral and psychological symptoms of dementia. BMJ. 2015; 350:h369.

7. Jones J, Sim TF, Hughes J. Pain assessment of elderly patients with cognitive impairment in the emergency department: implications for pain management - a narrative review of current practices. Pharmacy (Basel). 2017;5(2):E30.

8. Kaasalainen S. Pain assessment in older adults with dementia: using behavioral observation methods in clinical practice. J Gerontol Nurs. 2007;33(6):6-10.

9. Selbaek G, Kirkevold O, Engedal K. The prevalence of psychiatric symptoms and behavioural disturbances and the use of psychotropic drugs in Norwegian nursing homes. Int J Geriatr Psychiatry. 2007;22(9): 843-849.

10. Banerjee S. The Use of Antipsychotic Medication for People with Dementia: Time for Action. 2009. Available from: https:/www.dh.gov. uk. Accessed April 17, 2014.

11. Mahoney FI, Barthel DW. Functional evaluation: the Barthel index. Md State Med J. 1965;14:61-65.

12. Qureshi K, Hodkinson M. Evaluation of a 10 question mental test of the institutionalized elderly. Age Ageing. 1974;3(3):152-157.

13. Foroughan M, Wahlund LO, JafariZ, Rahgozar M, Farahani IG, Rashedi V. Validity and reliability of Abbreviated Mental Test Score (AMTS) among older Iranian. Psychogeriatrics. 2017;17(6):460-465.

14. Abbey J, De Bellis A, Piller N, et al. Funded by the JH \& JD Gunn Medical Research Foundation 1998-2002. Available from: www.prc. coh.org. Accessed January 2, 2018.

15. Abbey J, Piller N, De Bellis A, et al. The Abbey pain scale: a 1-minute numerical indicator for people with end-stage dementia. Int J Palliat Nurs. 2004;10(1):6-13.

16. Cohen-Mansfield J. Agitated behaviors in the elderly. II. Preliminary results in the cognitively deteriorated. J Am Geriatr Soc. 1986;34(10): $722-727$.

17. Finkel SI, Lyons JS, Anderson RL. Reliability and validity of the Cohen-Mansfield Agitation Inventory in institutionalized elderly. Int J Geriatr Psychiatry. 1992;7(7):487-490.

18. Husebo BS, Ballard C, Fritze F, Sandvik RK, Aarsland D. Efficacy of pain treatment on mood syndrome in patients with dementia: a randomized clinical trial. Int J Geriatr Psychiatry. 2014;29(8):828-836.
19. WHO Collaborating Centre for Drug Statistics Methodology. Guidelines for ATC classification and DDD assignment 2013. Oslo, Norway: WHO Collaborating Centre for Drug Statistics Methodology; 2012.

20. Office for Registration of Medicinal Products for Medical Devices and Biocidal Products. Available from: https://leki.urpl.gov.pl. Accessed January 2, 2018.

21. Closs SJ, Barr B, Briggs M. Cognitive status and analgesic provision in nursing home residents. Br J Gen Pract. 2004;54(509):919-921.

22. Monroe TB, Misra SK, Habermann RC, Dietrich MS, Cowan RL, Simmons SF. Pain reports and pain medication treatment in nursing home residents with and without dementia. Geriatr Gerontol Int. 2014; 14(3):541-548.

23. Husebo BS, Ballard C, Sandvik R, Nilsen OB, Aarsland D. Efficacy of treating pain to reduce behavioural disturbances in residents of nursing homes with dementia: cluster randomised clinical trial. BMJ. 2011; 343:d4065.

24. Neumann-Podczaska A, Nowak T, Suwalska A, et al. Analgesic use among nursing homes residents, with and without dementia, in Poland. Clin Interv Aging. 2016;11:335-340.

25. Sandvik R, Selbaek G, Kirkevold O, Aarsland D, Husebo BS. Analgesic prescribing patterns in Norwegian nursing homes from 2000 to 2011 : trend analyses of four data samples. Age Ageing. 2016;45(1):54-60.

26. Olsen BF, Rustøen T, Sandvik L, Jacobsen M, Valeberg BT. Results of implementing a pain management algorithm in intensive care unit patients: The impact on pain assessment, length of stay, and duration of ventilation. J Crit Care. 2016;36:207-211.

27. Norton MJ, Allen RS, Snow AL, Hardin JM, Burgio LD. Predictors of need-driven behaviors in nursing home residents with dementia and associated certified nursing assistant burden. Aging Ment Health. 2010; 14(3):303-309.

28. Buffum MD, Miaskowski C, Sands L, Brod M. A pilot study of the relationship between discomfort and agitation in patients with dementia. Geriatr Nurs. 2001;22(2):80-85.

29. Ahn $\mathrm{H}$, Horgas A. The relationship between pain and disruptive behaviors in nursing home residents with dementia. BMC Geriatr. 2013;(13):14.

30. Ballard C, Hanney ML, Theodoulou M, et al; DART-AD investigators The dementia antipsychotic withdrawal trial (DART-AD): long-term follow-up of a randomised placebo-controlled trial. Lancet Neurol. 2009;8(2):151-157.

31. Kupeli N, Vickerstaff V, White N, et al. Psychometric evaluation of the Cohen-Mansfield Agitation Inventory in an acute general hospital setting. Int J Geriatr Psychiatry. 2018;33(1):e158-e165.

32. Tan EC, Visvanathan R, Hilmer SN, et al. Analgesic use, pain and daytime sedation in people with and without dementia in aged care facilities: a cross-sectional, multisite, epidemiological study protocol. BMJ Open. 2014;4(6):e005757.

33. Habiger TF, Flo E, Achterberg WP, Husebo BS. The interactive relationship between pain, psychosis, and agitation in people with dementia: results from a cluster-randomised clinical trial. Behav Neurol. 2016; 2016:7036415.
Clinical Interventions in Aging

\section{Publish your work in this journal}

Clinical Interventions in Aging is an international, peer-reviewed journal focusing on evidence-based reports on the value or lack thereof of treatments intended to prevent or delay the onset of maladaptive correlates of aging in human beings. This journal is indexed on PubMed Central, MedLine,

\section{Dovepress}

CAS, Scopus and the Elsevier Bibliographic databases. The manuscript management system is completely online and includes a very quick and fair peer-review system, which is all easy to use. Visit http://www.dovepress. com/testimonials.php to read real quotes from published authors. 